\title{
Knowledge sharing and self- efficacy in an EFL context: the mediating effect of creativity
}

Farshad Parhamnia

Department of Knowledge and Information Science, Kermanshah Branch, Islamic Azad University, Kermanshah, Iran, and

Majid Farahian and Yusef Rajabi

Department of ELT, Kermanshah Branch, Islamic Azad University, Kermanshah, Iran

https://www.emerald.com/insight/content/doi/10.1108/GKMC-03-2021-0040/full/html 